\title{
28 Research Square \\ Frequency of Fabry Disease in a Juvenile Idiopathic Arthritis Cohort
}

\author{
Luciana Paim-Marques \\ University of Florida
}

Amanda Virginia Cavalcante

Escola de Saude Publica do Ceara

Islane Verçosa

Hospital Geral de Fortaleza

Paula Carneiro

Hospital Geral de Fortaleza

Marcia Souto-Maior

Universidade de Fortaleza

\section{Erlane Marques}

Hospital Infantil Albert Sabin

Simone Appenzeller ( $\nabla$ appenzellersimone@gmail.com )

Department of Medicine, Faculty of Medical Science, State University of Campinas, Cidade Universitária, Campinas SP, Brazil, CEP 13083-970; FAX: +55 19 3289-1818 https://orcid.org/0000-0001-5075-4474

\section{Research article}

Keywords: Fabry disease, Chronic Arthritis, Juvenile Idiopathic Arthritis, acroparesthesis, intronic variants

Posted Date: September 4th, 2020

DOI: https://doi.org/10.21203/rs.3.rs-60936/v1

License: (c) (i) This work is licensed under a Creative Commons Attribution 4.0 International License. Read Full License 


\section{Abstract}

Background. Fabry disease (FD) is a rare, X-linked, multisystemic lysosomal storage disorder (LSD) that

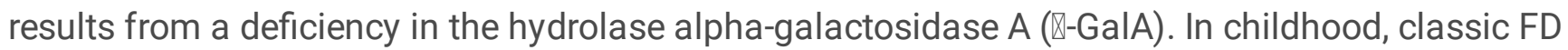
symptomatology is rare. Majority of children present with mild non-specific symptoms, including the musculoskeletal system. The prevalence of FD among juvenile idiopathic arthritis (JIA) patients is unknown.

Objective. The aim was to identify the frequency of FD in a JIA cohort, characterizing early clinical symptoms, enzyme titers and GLA genotyping.

Methods. Children with JIA followed in tertiary Children Hospital cohort were selected. Clinical, laboratorial, and familiar information was recorded. Molecular genetic testing to detect $G L A$ gene mutations was performed in the girls and enzymatic analysis in the boys.

Results. In 89 patients (56.2\% female, age at disease onset: $8.93 \pm 4.35$ years), one male (1.12\%) patient presented pathogenic mutation in GLA gene, $c .1244 T>C p . L 415 P$, one female patient had a variant of uncertain significance $c .38 \mathrm{C}>\mathrm{T}$ (p.Ala13Val). The enzymatic activity of alpha galactosidase was slightly decreased in 3 additional (3.4\%) patients. We observed the presence of intronic variants in $44.44 \%$ of our cohort: $c .1000-22 C>T ; c .370-81 \_-77 d e l ; c .640-16 A>G ; c .10 C>T ; c .548-125 C>G$ and $c .-12 G>A$. These variants and their combination were associated with clinical symptoms in our cohort.

Conclusions: The incidence of FD in our cohort was $1.12 \%$. Intronic variants were associated with symptomatology described in the literature. Screening for FD in JIA may be a reasonable strategy for those with atypical pattern pain.

\section{Background}

Fabry disease (FD) is a rare, $\mathrm{X}$-linked, multisystemic lysosomal storage disorder (LSD) that results from a deficiency in the hydrolase alpha-galactosidase $\mathrm{A}(\mathbb{Q}-\mathrm{Gal} \mathrm{A})$ caused by a GLA gene mutation. Its birth prevalence is estimated at 1:40.000-170.000(1). Recently, a neonatal screening has found a higher incidence of FD: 1:3.100 in Italy and 1:1.500 among males in Taiwan $(2,3)$.

The recessive $X$-linked characteristic of this disease makes male patients more affected, and female patient carriers or mildly affected by $\mathrm{X}$ inactivation. These differences influence the diagnostic methods (male patients have the enzyme dosage, and female patients should go straight to the genetic test), as well as clinical signs and life expectancies.

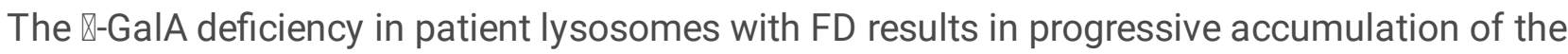
glycosphingolipid globotriaosylceramide (Gb3) in cells of many organ systems resulting in a chronic inflammatory process. (4). FD should be suspected in individuals presenting with acroparesthesias or other classic manifestations such as angiokeratomas, gastrointestinal symptoms, exercise intolerance, ocular abnormalities (cornea verticillata), decreased sweating, renal and cardiac involvement. Central nervous system presents with transient ischemic attacks and strokes predominantly in the vertebrobasilar system. 
However, FD has its onset in childhood with mild non-specific symptoms affecting frequently the musculoskeletal system. Peripheral neuropathic pain, fever, arthritis, and elevated erythrocyte sedimentation rate (ESR) can be observed (5-8).

High disease suspicion and screening in high-risk patients is a cost-effective strategy for identifying FD patients (10). Since musculoskeletal features are frequently observed in FD, and juvenile idiopathic arthritis (JIA) is the most frequent chronic, inflammatory arthritis disorder observed in childhood, this study aimed to identify the frequency of FD in a JIA cohort by characterizing early clinical symptoms, enzyme titers and GLA genotyping.

\section{Materials And Methods}

Consecutive JIA patients, classified according to ILAR criteria(9), followed in the pediatric rheumatology outpatient clinic at Albert Sabin Children's Hospital were invited to participate in this cross-sectional study from December 2014 to December 2017. Of a total of 180 JIA patients followed during this period, 89 (49.4\%) agreed to join this study.

The local ethics committee approved this study (Albert Sabin Childhood Hospital, Fortaleza, Ceará, Brazil, CAAE: 37270414.0.0000.5042). All patients (and legal representatives if children under 18 years) included signed the informed consent and assent form.

\section{JIA history}

The following demographic and disease characteristics were obtained through careful chart review for each patient: age, sex, age at disease-onset, JIA subtype, articular, and extra-articular manifestations. The following immunological features were retrieved from medical charts: rheumatoid factor (RF) by latex agglutination test, antinuclear antibodies (ANA) by indirect immunofluorescence assay (IIFA) on Human epithelial type 2 (HEp-2 cells), anti- ds DNA, anti-Smith, anti-RNP, anti-SSa, anti-SSb, anti-cardiolipin antibodies by enzymelinked immunosorbent assay (ELISA), and human leukocyte antigen (HLA) B27 by polymerase chain reaction (PCR).

\section{Study questionnaire}

JIA patients were inquired about FD features through a structured questionnaire based on early signs and symptoms, applied by the treating physician (11). This questionnaire contains queries about clinical symptoms (heat intolerance, hypo/hyperhidrosis, angiokeratomas, telangiectasias, chronic fatigue, abdominal distension, dyspepsia, diarrhea, gastric fullness sensation, weight gain difficulty, tinnitus, dysacusis, acroparesthesia), physical exam findings (telangiectasia, angiokeratoma), past medical history (stroke and transient ischemic attack), and familial history (stroke, transient stroke, sudden death, end-stage renal or FD).

\section{$\underline{\text { Clinical evaluation }}$}


All patients were evaluated by a board-certified pediatric rheumatologist. All patients had a complete clinical, osteoarticular and neurological exam performed at study entry.

JIA patients underwent a complete ophthalmological examination by a board-certified pediatric ophthalmologist. Positive findings were reviewed by a second board-certified ophthalmologist. Refractive errors were measured by a hand-held auto-refractor keratometer retinomax $\mathrm{K}$ plus 2 . The best-corrected visual acuity of each eye was determined by Snellen letters and numbers chart, measured at a distance of 6 meters for children above 6 years. Non-literate children's visual acuity was evaluated by the Snellen E and Snellen number chart at an appropriate distance. Anterior segment (cornea, iris, and lens crystalline) was evaluated by slit-lamp examination. The optic nerve, macula, and posterior pole vessels were analyzed with direct ophthalmoscopy. Intraocular pressure (IOP) was measured by applanation with the Perkins tonometer method and was considered normal if ranged from $10-20 \mathrm{mmHg}$. A tear breakup time (TBUT) test was performed after placing a drop of fluorescein in the cul-de-sac in order to determine keratoconjunctivitis. The presence of cornea verticillata was evaluated by a board-certified pediatric ophthalmologist during complete ophthalmological evaluation.

The patients were also evaluated through a transthoracic 2D echocardiogram (Echo), and conduction disturbance was evaluated through 12 channels electrocardiogram (ECG) analyzed by a board-certified pediatric cardiologist.

Kidney involvement was evaluated through serum creatinine, 24-hour urinary microalbuminuria, and urinary sodium.

\section{Genetic testing}

The genetic test was sponsored by Shire and carried out in the outpatient clinic by a trained nurse. Blood was drawn after re-authorization by the patients and legal representatives at an outpatient clinic and placed at 5 blood spots on filter paper duly identified with the patient's, doctor's and nurse's data.

For males, an initial screening of the $₫$-GalA enzyme and the acidic sphingomyelinase (control) enzyme activity and measurement of globotriaosylsphingosine (lyso-Gb3) by high-performance liquid chromatography (HPLC) was performed by tandem mass spectrometry at Centogene (Germany).

For women or male patients with abnormal enzyme activity, the analysis of the GLA gene (ref: NM_000169.2) was conducted by PCR and sequencing of the entire coding region and the highly conserved exon-intron splice junctions. This test has been developed and validated by Centogene AG for clinical purposes. Patients

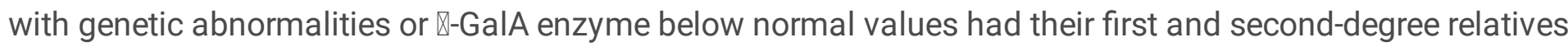
(parents, grandparents, and siblings), when possible, screened for FD with appropriate genetic investigation (enzyme levels or gene identification), and referred to genetic monitoring.

\section{Statistical Analyses}

All statistical analysis was performed using SPSS 20.0 software package. Results are shown in absolute number and percentage or mean and standard deviation (SD). Chi-square or Fischer exact test was used to 
compare categorical variables. The continuous variables were compared by analysis of variance (ANOVA). A $p$-value $\leq 0.05$ was considered clinically significant.

\section{Results}

A total of $89 \mathrm{JIA}$ patients (mean age of $15.80 \pm 3.95)$ were included. A predominance of women $(56.17 \%)$ and pauciarticular subgroup (47.2\%) was observed. The mean age of disease onset was $8.93 \pm 4.35$ years. The subtype classification, clinic, laboratory abnormalities of JIA patients were summarized in Tables 1 and 2 . We did not observe significant differences in patient demographics and clinical characteristics between those who were enrolled in the study and those who did not agree to participate (data not shown). The patients were informed the study was about Fabry disease, but the clinic survey was done along with the consent, avoiding researches, and decreasing the likelihood of falsely elevated prevalence of symptoms suggestive of FD.

The genetic tests were performed in all (56.17\%) female patients, while enzyme activity was performed in 39 $(43.82 \%)$ males of our cohort. The results identified $4(4.49 \%)$ males with decreased enzyme activity. For that reason, we had a total of $54(60.67 \%)$ patients with genetic tests done.

One of 89 (1.12\%),patient (male, admixture of Caucasian and Native South American (Indian), pauciarticular

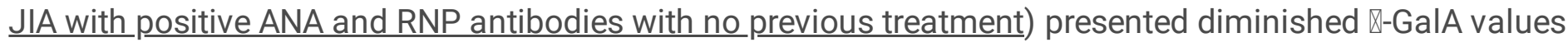
and abnormal lyso-Gb3 levels. His genetic test showed the GLA variant c.1244T>C p.L415P (Ref: Serebrinsky, 2006) confirming FD (12). That patient presented hands and feet pain at the age of 5 years, associated with sporadic low-grade fever, especially after exercises, and significant anhidrosis. At the age of eleven, he reported bilateral ankle pain and swelling. He was referred to Pediatric Rheumatology for evaluation. The chronic ankles arthritis was clinically confirmed, however, the complaint of absence of sweat, fever after activities, burning_pain, abdominal pain, some of them uncommon in JIA set of symptoms, raised the hypothesis of JIA. His physical exam and workup showed presence of angiokeratomas around the belly. bottom, cardiac abnormalities, and cornea verticillata. His pedigree was rich for strokes, heart attack and, transient ischemic attack in family members under the age of 50. This patient also had intronic GLA variants, c.370-81_-77del (rs5903184) on intron 2, c.640-16A> G (rs2071397) on intron 4, c.1000-22C> T (rs2071228) on intron 6 e, c. $-10 C>T$ (rs2071225) in region 5'UTR exon 1. Once he started the enzyme replacement, his symptoms improved: hypohidrosis, abdominal pain, dyspepsia, heat intolerance, acroparesthesias, and angiokeratomas. However, no changes in the cornea verticillata and arthritis was observed. Magnetic resonance imaging of the right ankle showed increased synovial fluid and synovial thickening. Methotrexate $\left(15 \mathrm{mg} / \mathrm{m}^{2} /\right.$ week) was added to his therapeutic plan with subsequent resolution of his synovitis.

Another female pauciarticular JIA patient presented a previously unreported heterozygous variant in exon 1 of the GLA gene $c .38 C>T$ p.Ala13Val (No reference). This variant is located in a non-conserved nucleotide, and a frankly conserved amino acid position, with small physical-chemical difference between the amino acid alanine and valine (Alamut v.2.4). Polyphen-2, SIFT and MutationTaster analysis predict this variant as probably benign. The patient also presented the following intronic GLA variants, c.370-81_-77del (rs5903184) on intron 2, c.640-16A> G (rs2071397) on intron 4, c.1000-22C> $T$ (rs2071228) at intron 6 e, c.-10C>T (rs2071225) in region 5'UTR exon 1. Clinically, cornea verticillata was observed on her ophthalmologic exam. 
She denied any other symptoms related to FD, with early stroke history in her mother with negative genetic test.

A third pauciarticular JIA female presented a variant on GLA gene exon 1 heterozygous C.48T>G p.Leu16Leu (rs201449986) considered benign due to the change of the same codons leucine for leucine, and because it is not located in a splicing sequence. This variant was recently checked in 3 genetic banks: Online Mendelian Inheritance in Man (OMIM), Clinvar and HGNC (HUGO Gene Nomenclature Committee) and is still described as neutral. The statistical association of this variant with FD clinical symptoms, laboratory and GLA variants are described on Table 3.

The enzymatic activity of $\rrbracket-G a l A$ was slightly decreased in 3 (3.4\%) additional patients with a total of four (4,49\%) abnormal enzymatic essay. Two patients had history and clinical symptoms suggestive of FD (hypohidrosis, acroparesthesia and dy spepsia, weight gain difficulty, familial history of sudden death and end-stage renal disease). The third one had no symptoms or family history suggestive of FD. All three patients had negative genetic test for FD.

In addition, we identified a total of 18 (22.90\%) patients with cardiac abnormalities, 10 (52.63\%) patients had right bundle branch block, 4 (20\%) presented mitral valve prolapse, 3 (15.78\%) had valvar regurgitation. We also observed 3 (15.78\%) patients with ventricular hypertrophy; one systemic JIA patient with right ventricular hypertrophy secondary to pulmonary hypertension, one polyarticular JIA patient with left ventricular hypertrophy, and our index patient with a left concentric ventricular hypertrophy.

A total of $54(60.67 \%)$ patients had genetic testing. Of these, 26 (48.14\%) patients had GLA variants (intronic and exonic). In the majority of the patients (92.30\%), multiple intronic GLA mutations were observed.

The most observed intronic variants were: 24 (92.30\%) of the 26 showed $c .1000-22 C>T$ (rs2071228) intron 6,

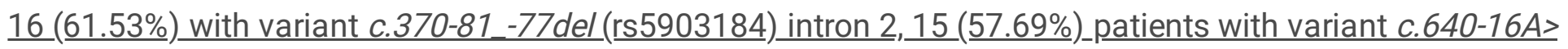
$\underline{G}$ (rs2071397) intron 4; 8 (30.76\%) of them with c. 10C>T (rs2071225) in 5úTR exon 1; 7 (26.92\%).patients

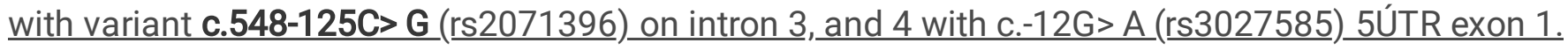

We also observed presence of complex intronic haplotypes $(\underline{\mathrm{ClH}})$ in $44.44 \%$ of the total of tests performed. The intronic variants as well as the $\mathrm{CIH}$ had positive correlations with FD symptoms (Table 3 ). They were grouped as Haplotype 1- c. $10 C>T$, c. 370-77_-81del, c. 640-16A>G, c. 1000-22C>T in 8 (14.81\%), Haplotype 2c.370-77_-81del, c. 640-16A>G, c. 1000-22C>T in 7 (12.97\%), Haplotype 3- c. 548-125C>G, C. 1000-22C>T, c.-12G>A in 4 (7.40\%), ; Haplotype 4- c.548-125C>G, c. 1000-22C>T in 2 (3.70\%), Haplotype 5- c.370-81_-77del,

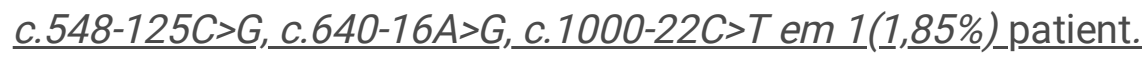

\section{Discussion}

We observed a frequency of $1.12 \%$ of FD in our cohort. There are no epidemiological studies of FD in Brazil, however, the frequency expected for live male births in the general population $0.0025 \%(p=0.0088)(13)$. In the pediatric population, a Portuguese group studied a cohort of 292 patients with JIA and its association of $\underline{F D}$, however, they did not find a classic pathogenic mutation (10). 
The ethnicity of the affected child [admixture of Caucasian and Native South American (Indian)] $\underline{\text { is not }}$ included in the populations of higher incidence for FD $(\underline{2}, \underline{3})$.

In this cohort three exonic GLA variants were found. The first variant, $C .1244 T>C$, was in our index case described as pathogenic and referenced by Serebrinsky et al in 2006 as disease-causing according to ACMG variant classification recommendations (17).

The second variant ( $c .38 \mathrm{C}>T$ p.Ala $13 \mathrm{Val}$ ) never been described before, and It is located in a non-conserved nucleotide and weakly conserved amino acid position, with small physicochemical differences between the amino acids alanine and valine (Alamut v.2.4). Software analyses by Polyphen-2, SIFT, MutationTaster and Align-GVGD predict this variant is probably benign according to ACMG recommendations of interpretation of sequence variations (17).

The third variant in GLA exon 1 c.48T>G (rs201449986) was considered likely benign (17). This abnormal sequence is not expected to have clinical significance because it does not alter an amino acid residue and is not located within the splice consensus sequence. According to Sequence Project (http://evs.washington.ed/EVS/;). This allele frequency is $1 / 6728$.

Patients with classic FD have no residual or around 30-35\% of $\otimes-G a l A$ activity enzyme (14).

For diagnosis, is required increased levels of Gb3 in lysosomes (15). The inheritance is X-linked and recessive, which means that female heterozygous genotype presents with incomplete penetrance, due to $\mathrm{X}$ inactivation. The mildest disease allows women to have residual enzyme activity; for that reason, the genetic analysis is the gold standard for diagnosis (16). The enzymatic activity can be measured in peripheral blood cells or dry blood spots. In our study, all analysis was done with dry blood spots. The enzymatic activity is very variable among FD patients, and among different organs (18). This variations are a challenge to establish thresholds of FD pathogenicity (18).

In this study, we identified 4 (4.5\%) patients with decreased levels of a-GalA activity in the plasma. Three children had a family history and symptoms suggestive of FD. Only one patient with decreased levels of the enzyme activity in the plasma was asymptomatic.

A defective $\triangle$-GalA leads to accumulation of undegraded substrates [globotriaosylceramide and globotriaosylsphingosine (Gb3 and Lyso Gb3)] inside lysosomes, acting as damage-associated molecular patterns (DAMPs) or stimulating DAMP production, activating an inflammatory pathway, inducing apoptosis and a toll-like receptor- 4 (TLR4) mediated innate immune system pro-inflammatory cytokines secretion (IL-1 $\beta$ and TNF-a) (19). These different cellular mechanisms contribute to the different phenotypic expression of FD (20). These cytokines secretion are a characteristic trace of autoinflammatory disorder(21). The recognition of Gb3 or lyso-Gb3 as antigens also influence the invariant natural killer T cell (iNKTs) to induce the release of others inflammatory cytokines such as interferon-gamma (IFN- $-\gamma)$, tumor necrosis factor-alpha (TNF-a), interleukins (IL): IL-4, IL-5, IL-9, IL10, IL13, and IL-17. This inflammatory cascade produces a continuous stimulus responsible for the induction and maintenance of the autoimmune response. The activation of the above mentioned inflammatory pathway explains the presence of autoimmune and autoinflammatory 
features in FD. Our index patient had, beyond the classic FD symptoms, a positive ANA and RNP antibodies, and chronic oligoarthritis (bilateral ankles).

The high frequency of cardiac involvement in our cohort (Table 2) is probably related to JIA, which can involve all of the cardiac structures, including pericardium, myocardium, endocardium; coronary vessels; valves and conduction system (22). However, FD also causes cardiac abnormalities including conduction abnormalities, valvular dysfunction, arrhythmias in childhood, progressing to ventricle concentric hypertrophy in non-treated patients (23). Our index patient had mitral prolapse with reflux and left ventricle concentric hypertrophy, cardiac manifestations frequently observed in FD.

The main musculoskeletal symptoms described in early FD is acroparesthesia, however, chronic inflammatory joint and bone diseases (polyarticular, oligo and monoarticular, gout, osteoporosis), degenerative, neurologic arthropathy (Charcot's foot)(7), Heberden-like nodules (24)and also myositis have been described (8). Nowadays, the coexistence of FD and autoimmune disease has gained increased visibility in the medical literature, and patients with FD and systemic lupus erythematosus $(25,26)$ rheumatoid arthritis(27), autoimmune hypothyroidism (28), Ig A nephropathy (29) and granulomatosis with polyangiitis (30) have been described. Patients with FD and rheumatic manifestations have a significant delay in FD diagnosis that can last up to 16 years or more (31). The most common associated mutations observed in FD patients presenting with rheumatic manifestations were R118C and A143T (31).

Other interesting finding in this study was the presence of GLA intronic mutations in patients with JIA. Six intronic variants were identified, c. 370-81_-77del, c. 548-125C $>G$ and c.548-162A>T c.640-16A>G.c. 1000$22 C>T, C .-10 C>T$ and $c .-12 G>A$ located in introns $2,3,4,6$ and in the 5'UTR region of GLA exon 1 , respectively. Three single nucleotide polymorphisms (SNP)_[C.37081_-77del (rs5903184), c. $-12 G>A$ (rs3027585) and c.-10C>T(rs2071225)] are relatively common to different ethnic groups, with a frequency of the minor allele about $10 \%$ in the British population (32), and $12 \%$ in Latin populations (OMIM). These SNPs are common to the Portuguese population, the greatest ancestor of the Brazilian citizens ( $(\underline{33})$. Those variants were found in 12 (22.22\%) c. 370-81_-77del, 8 (14.81\%) c. $-10 C>T$ and 4 (7.40\%) $c .-12 G>A$ in the 54 tested patients.

As the previous variants, other SNPs and its combination, denominated $\mathrm{ClH}$, have been described as associated with Fabry-similar symptoms.

The two SNPs of the a-GalA gene c.1000-22C > T [rs2071228] and c.640-16A>G (rs2071397) were associated with the presence of angiokeratomas and acroparesthesias in patients with hypertrophic cardiomyopathies without FD (34). In vitro and in vivo analyzes have shown that polymorphisms in the 5'UTR region can alter the expression of the a-GalA gene, with possibleclinical relevance, particularly in male patients with $G L A$ variants associated with a high reduction in enzyme activity_(35). The c. $-10 T$ allele, found in $15 \%$ of the positive results of this study, was previously associated with a decrease in a-GalA activity in leukocytes (33), and has a possible correlation with neurological injuries such as stroke, transient ischemic attack, white matter injury and fine fiber neuropathy, in patients with peripheral neuropathy_(36), as well as in patients with $\underline{F D}(\underline{35})$. In our study, this variant was associated with the difficulty of weight gain and ocular changes. 
Classic ocular manifestations in FD are observed by age 4, while in heterozygotes present it later, around the age 10 (37).

The c.-10T allele, located in the $5^{\prime}$ non-coding region, has been associated with a decrease in the expression of a-GalA (34), altering in the promoter gene the nuclear protein binding site (38). Studies are still needed to determine the real role of this variant in GLA. Recent data suggested that reduced enzyme activity, with normal levels, may be a risk factor in Parkinson's disease (39). There are numerous descriptions of the $c .-10 T$ allele and Fabry-simile manifestations (34).

The most frequent intronic variant in our cohort was c. 1000-22C>T(rs2071228) 24 (44.44\%) of 54 patients positive for genetic alterations. This variant, located in intron 6 , is phenotypically associated with Fabry, and idiopathic hypertrophic cardiomyopathy by the bank Vep Ensembl. This allele is also associated with some $\underline{\mathrm{CIH}}$ that seem to translate enzymatic alteration with the accumulation of glycosphingolipids (38). Haplotypes are a combination of inherited alleles at adjacent loci. There are numerous reports of groups of alleles causing Fabry simile changes and Fabry disease per se. Gervas-Arruga et al, studied a ICH $(c .-10 C>T, c .369+$ 990C>A, c.370-81_370-77delCAGCC, c. 640-16A>G, C. 1000-22C> T) in the GLA gene. They evaluated the enzymatic levels in cells (fibroblasts and leukocytes) in the plasma and the enzyme's quantitative expression. The results suggested an altered expression pattern of the studied gene, but without sufficient abnormality of enzyme levels in plasma, leukocytes, and skin fibroblasts, however, with glycosphingolipids accumulation of in fibroblasts, renal and glomerular tubular cells (38).

Another study described a similar $\mathrm{CIH}$ on a GLA in a patient with the early systemic onset of FD. The patient carried only the haplotype $(-10 \mathrm{C}>T, c .370-77-81 \mathrm{del}$, c.640-16A>G, c. 1000-22C $>T)$, suggesting that those variants localized in a promoter and intronic regulatory region, could cause disease even without the presence of exonic abnormalities (40). In our cohort, we had $8(14.8 \%)$ of the 54 tests, that presented this same haplotype (-10C>T, c. 370-77-81del, c. 640-16A>G, c. 1000-22C>T), including the patient with FD and the patient with $c .38 C>T$ variant who presented cornea verticillata. Haplotypes 1 and 2 were associated with visual changes and corneal abnormalities, and haplotype 3 had a positive association with valve changes (table 1).

There was no association of acroparesthesias/peripheral neuropathies with $\mathrm{ICHs}$, despite its incidence in half of our sample. A limited number of genetic tests in our cohort may have influenced the possible positive associations between the variants found and reported clinical signs.

In our cohort, we observed a variety of clinical symptoms related to FD in the JIA cohort. Almost 50\% described acroparesthesias, and a third of the patients had difficulty of weight gain, while $42 \%$ had stroke familial history. All these features could be associated with chronic arthritis and its treatment. Our index patient was initially treated with enzyme replacement, considering FD was misdiagnosed with JIA. Despite a significant improvement of anhidrosis, muscular and abdominal pain and the GB3 levels, he had persistent chronic arthritis course with synovial thickening, reassuring for JIA. Methotrexate was initiated improving his symptoms. We showed another patient with a VOUS for FD, unfortunately, young age is an obstacle for built up identification FD or FD-like symptoms, and these patients need extensive follow-up to determine if these associated mutations are pathogenic or not, especially in females. 
We observed FD as a co-morbid in $1.12 \%$ of our JIA cohort but the small number of JIA patients in this cohort was a limitation for this study. We only included $50 \%$ of our cohort, mostly to logistic issue (missing appointments, incomplete clinical evaluation). The patient's legal representatives signed the informed consent and were therefore aware that the study was about FD. However, the clinic survey was done at the time of the consent, avoiding researches, and decreasing the likelihood of the data showing a falsely elevated prevalence of symptoms suggestive of FD in a JIA cohort.

Other limitation is the genetic test, which should have been done for all patients (males and females). Although we can deduce that patients with normal enzyme levels do not present exonic pathogenic changes, the same cannot be concluded from intronic mutations and GLA haplotypes, which could interfere in the $₫$ GalA activity levels in the future. We also had no access to their family medical records. Therefore, we could not explain the high incidence of familiar history of vascular events and confirm other possible confounders such as diabetes, obesity or antiphospholipid syndrome.

\section{Conclusions}

In our cohort, FD was present in $1.12 \%$ of JIA patients. FD may have autoimmune features, and a high index of suspicion is necessary for the diagnosis. Pediatricrheumatologists should be aware that FD could present with similar classic autoimmune disease features, but they can also co-exist. We need to be careful with those patients with JIA and "dysautonomia" symptoms (persistent extremities or diffuse pain, gastroparesis or abdominal pain, absence of sweat, and arrhythmias), and be more detail with the family history, looking for strokes, sudden death and heart attacks at a young ag $\underline{\text { e }(<50)}$, and kidney disease leading to transplant. Those questions are crucial for FD diagnosis, and early disease identification is an effective strategy to avoid kidney transplants and early death with the enzyme replacement $(\underline{10})$. In the future, we hope to perform enzyme activity and genetic tests in all suspected patients.

\section{List Of Abbreviations}

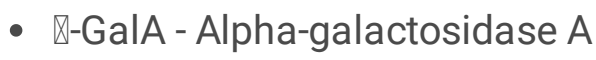

- ANA - antinuclear antibodies

- ANOVA - Analysis of variance

- $\mathrm{ClH}$ - Complex intronic haplotypes

- DAMPs - Damage-associated molecular patterns

- ds DNA - Double stranded deoxyribonucleic acid

- ECG - Electrocardiogram

- Echo - Echocardiogram

- ELISA - Enzyme-Linked Immunosorbent Assay

- ESR - Erythrocyte sedimentation rate

- FD - Fabry disease

- Gb3 - Globotriaosylceramide 
- HEp-2- Human epithelial type 2

- HGNC - HUGO Gene Nomenclature Committee

- HLA - Human leukocyte antigen

- HPLC - High-performance liquid chromatography

- IFN- - Interferon-gamma

- IIFA - immunofluorescence assay

- IL - Interleukins

- ILAR - International League against Rheumatism

- IOP - Intraocular pressure

- JIA - Juvenile idiopathic arthritis

- LSD - Lysosomal storage disorder

- Lyso-Gb3-globotriaosylsphingosine

- OMIM - Online Mendelian Inheritance in Man

- PCR - Polymerase Chain Reaction

- RF - rheumatoid factor

- RNP - Ribonucleoprotein

- SNP - Single nucleotide polymorphism

- TBUT - Tear breakup time

- TLR4 - Toll-like receptor- 4

- TNF-a - Tumor necrosis factor alpha

\section{Declarations}

- Ethics approval and consent to participate

- The local ethics committee approved this study (Albert Sabin Childhood Hospital, Fortaleza, Ceará, Brazil, CAAE: 0.0000.5042). All patients (and legal representatives if children under 18 years) included signed the informed consent and assent form.

- Consent for publication - Not applicable

- Availability of data and materials:

- All data generated or analysed during this study are included in this published article [and its supplementary information files].

- Conflict of Interest disclosure:

- All authors declare that they have no conflict of interest related to the study.

\section{- Funding}

- Fundação Apoio À Pesquisa Estado São Paulo-Brasil (FAPESP 2008/02917-0 and 2009/06049-6 and 2009/15286-1 and 2013/07559-3), 
- Conselho Nacional Pesquisa Desenvolvimento-Brasil CNPq 304255/2015-7 and 473328/2013-5 and 401477/2016-9),

- Coordenação de Aperfeiçoamento de Pessoal de Nível Superior - Brasil (CAPES) Finance Code 001

- Shire Brasil for genetic tests.

\section{- Authors' contributions - Please use the author's initials (Al, ZE, SA):}

- LPM, EM, SA- Substantial contributions to the conception or design of the work; or the acquisition, analysis, or interpretation of data for the work.

- LPM, AVC, IV, PC, MSM, SA- Drafted the work or revising it critically for important intellectual contente.

- All authors gave final approval of the version to be published.

- All authors agree to be accountable for all aspects of the work in ensuring that questions related to the accuracy or integrity of any part of the work are appropriately investigated and resolved.

\section{- Acknowledgements:}

- Aureliana Euclides and Fábia Sardinha.

Correspondence: Simone Appenzeller-Department of Medicine, Faculty of Medical Science, State University of Campinas, Cidade Universitária, Campinas SP, Brazil, CEP 13083-970; FAX: +55 19 3289-1818

Email: appenzel@unicamp.br

\section{Grants}

Simone Appenzeller received grants from Fundação Apoio À Pesquisa Estado São Paulo-Brasil (FAPESP 2008/02917-0 and 2009/06049-6 and 2009/15286-1 and 2013/07559-3), Conselho Nacional Pesquisa Desenvolvimento-Brasil CNPq 304255/2015-7 and 473328/2013-5 and 401477/2016-9), Coordenação de Aperfeiçoamento de Pessoal de Nível Superior - Brasil (CAPES) Finance Code 001

\section{References}

1. Biegstraaten M, Arngrímsson R, Barbey F, Boks L, Cecchi F, Deegan PB, et al. Recommendations for initiation and cessation of enzyme replacement therapy in patients with Fabry disease: The European Fabry Working Group consensus document. Orphanet Journal of Rare Diseases. 2015;10 (1):1-10. org/10.1186/s13023-015-0253-6.

2. Spada M, Pagliardini S, Yasuda M, Tukel T, Thiagarajan G, Sakuraba H, Ponzone A, Desnick RJ. High incidence of later-onset Fabry disease revealed by newborn screening. Am J Hum Genet. 2006;79:31-40. doi.org/1086/504601.

3. Chien YH, Lee NC, Chiang SC, Dobrovolny R, Huang AC, Yeh HY, Chao MC, Lin SJ, Kitagawa T, Desnick RJ, Hsu LW. Newborn screening for Fabry disease in Taiwan reveals a high incidence of the later-onset $G L A$ mutation c.936+919G>A (IVS4+919G>A). Hum Mutat. 2009;30(10):1397-1405. doi.org/1086/504601.

4. Brady RO, Gal AE, Bradley RM, Martensson E, Warshaw AL, Laster L. Enzymatic defect in Fabry's disease. Ceramidetrihexosidase deficiency. N. Engl. J. Med. 1967;276:1163-1167. 
org/10.1056/NEJM196705252762101.

5. Cimaz R, Guillaume S, Hilz MJ, Horneff G, Manger B, Thorne JC, Torvin Moller A, Wulffraat NM, Roth J. Awareness of Fabry disease among rheumatologists-current status and perspectives. Clinical Rheumatology. 2011;30(4):467-475. org/10.1007/s10067-010-1445-z.

6. Ivleva A, Weith E, Mehta A \& Hughes DA. The Influence of Patient-Reported Joint Manifestations on Quality of Life in Fabry Patients. JIMD Reports. 2018;41:37-45. doi.org/10.1007/8904_2017_84.

7. Thévenot C, Crrouzet J, Villiaumey J, Avouac B, Le Charpentier Y, Voisin MC. Les manifestations articulaires de la maladie de Fabry. A propos de deux observations. HÔP PARIS. 1992;486-493.

8. Chimenti C, Padua L, Pazzaglia C, Morgante E, Centurion C, Antuzzi D et al. Cardiac and skeletal myopathy in Fabry disease: A clinicopathologic correlative study. Human Pathology. 2012;43(9): 14441452. doi.org/10.1016/j.humpath.2011.09.020.

9. Petty RE, Southwoood TR, Baum J, Bhettay E, Glass DN, Manners P, Maldonado-Coco J, Suarez-Almazor M, Orozco-Alcala JPA. JIA criteria Article ILAR 1997. The Journal of Rheumatology. 1998;25:1991-1994.

10. Gonçalves MJ, Mourão AF, Martinho A, Simões O, Melo-Gomes J, Salgado M et al. Genetic Screening of Mutations Associated with Fabry Disease in a Nationwide Cohort of Juvenile Idiopathic Arthritis Patients. Frontiers in Medicine. 2017;4(12):1-5. org/10.3389/fmed.2017.00012.

11. Sestito S, Ceravolo F \& Concolino D. Anderson- Fabry Disease in Children. Current Pharmaceutical Design.2013;19:6037-6045. doi.org/10.2174/13816128113199990345.

12. Serebrinsky GP, Pascucelli V, Politei JM. Gene symbol: GLA. Disease: Fabry disease. Hum Genet. 2006;119:361. PMID: 17230649.

13. Matern D, Gavrilov D, Oglesbee D, Raymond K, Rinaldo P \& Tortorelli S. Newborn screening for lysosomal storage disorders. Seminars in Perinatology. 2015;39: 206-216. doi.org/10.1053/j.semperi.2015.03.005

14. Chien YH, Lee NC, Chiang SC, Desnick RJ \& Hwu WL. Fabry Disease: Incidence of the Common LaterOnset a-Galactosidase A IVS4+919G $\rightarrow$ A Mutation in Taiwanese Newborns- Superiority of DNA-Based to Enzyme-Based Newborn Screening for Common Mutations. Molecular Medicine. 2012;18(5):780-784. org/10.2119/molmed.2012.00002.

15. Desnick RJ, Allen KY, Desnick SJ, Raman MK, Bernlohr RW, Krivit W. Fabry's disease: enzymatic diagnosis of hemizygotes and heterozygotes. Alpha- galactosidase activities in plasma, serum, urine, and leukocytes. J Lab Clin Med. 1973;81:157-171. PMID: 4683418.

16. Pagnini I, Borsini W, Cecchi F, Sgalambro A, Olivotto I, Frullini A, et al. Distal extremity pain as a presenting feature of Fabry's disease. Arthritis Care Res (Hoboken). 2011;63:390-395. org/10.1002/acr.20385.

17. Richards CS, Bale S, Bellissimo DB, et al. ACMG recommendations for standards for interpretation and reporting of sequence variations: Revisions 2007. Genet Med. 2008;10(4):294-300. doi:10.1097/GIM.0b013e31816b5cae.

18. Havndrup O, Christiansen M, Stoevring B, Jensen M, Hoffman-Bang J, Andersen PS, et al. Fabry disease mimicking hypertrophic cardiomyopathy: Genetic screening needed for establishing the diagnosis in women. European Journal of Heart Failure. 2010;12(6): 535-540. doi.org/10.1093/eurjhf/hfq073. 
19. De Francesco PN, Mucci JM, Ceci R, Fossati CA \& Rozenfeld PA. Fabry disease peripheral blood immune cells release inflammatory cytokines: Role of globotriaosylceramide. Molecular Genetics and Metabolism. 2013;109(1):93-99. doi.org/10.1016/j.ymgme.2013.02.003.

20. Aerts JM, Groener JE, Kuiper S, Donker-Koopman WE, Strijland A, Ottenhoff R et al. Elevated globotriaosylsphingosine is a hallmark of Fabry disease. Proceedings of the National Academy of Sciences of the United States of America. 2008;105(8): 2812-2817. org/10.1073/pnas.0712309105.

21. Jesus AA, Canna SW, Liu Y \& Goldbach-Mansky R. Molecular Mechanisms in Genetically Defined Autoinflammatory Diseases: Disorders of Amplified Danger Signaling. Annu Rev Immunol. 2015;33:823874. org/10.1146/annurev-immunol-032414-112227.

22. Koca B, Sahin S, Adrovic A, Barut K, Kasapcopur O. Cardiac involvement in juvenile idiopathic arthritis. Rheumatol Int. 2017;37(1):137-142. doi:10.1007/s00296-016-3534-z)

23. Germain DP, Fouilhoux A, Decramer S, Tardieu M, Pillet P, Fila M, Rivera S, et al. Consensus recommendations for diagnosis, management and treatment of Fabry disease in paediatric patients. Clin Genet. 2019;96(2):107-117. doi:10.1111/cge.13546.

24. Lidove O, Zeller V, Chicheportiche V, Meyssonnier V, Sené T, Godot S \& Ziza J M. Musculoskeletal manifestations of Fabry disease: A retrospective study. Joint Bone Spine. 2016;83(4): 421-426. org/10.1016/j.jbspin.2015.11.001.

25. Rahman P, Gladman DD, Wither JSM. Coexistence de Fabry's disease and Systemic Lupus Erythematous. Clinical and Experimental Rheumatology. 1998;16:475-478. PMID: 9706432.

26. Chatre C, Filippi N, Roubille F \& Pers Y-M. Heart Involvement in a Woman Treated with Hydroxychloroquine for Systemic Lupus Erythematosus Revealing Fabry Disease. The Journal of Rheumatology. 2016;43(5):997-998. doi.org/10.3899/jrheum.150726.

27. Martinez P, Aggio M \& Rozenfeld P. High incidence of autoantibodies in Fabry disease patients. Journal of Inherited Metabolic Disease. 2007;30(3):365-369. doi.org/10.1007/s10545-007-0513-2.

28. Katsumata N, Ishiguro A \& Watanabe H. Fabry disease superimposed on overt autoimmune hypothyroidism. Clinical Pediatric Endocrinology. 2011;20(4):95-98. doi.org/10.1297/cpe.20.95.

29. Yin G, Wu Y, Zeng CH, Chen HP \& Liu ZH. Coexistence of Fabry disease and IgA nephropathy: a report of two cases. Irish Journal of Medical Science. 2014;183(4): 671-675. doi.org/10.1007/s11845-014-1161-9

30. Hanaoka H, Hashiguchi A, Konishi K, Ishii T \& Kuwana M. A rare association between Fabry's disease and granulomatosis with polyangiitis: A potential pathogenic link. BMC Nephrology. 2014;15(1): 1-5. https://doi.org/10.1186/1471-2369-15-157.

31. Rosa Neto NS, Bento J \& Pereira R. Higher rate of rheumatic manifestations and delay in diagnosis in Brazilian Fabry disease patients. Advances in rheumatology. 2020;60(1), 7. doi:10.1186/s42358-0190111-7.

32. Davies JP, Winchester BG \& Malcolm S. Sequence variations in the first exon of alpha-galactosidase A. Journal of medical genetics. 1993;30(8),658-663. https://doi.org/10.1136/jmg.30.8.658.

33. Oliveira JP, Ferreira S, Reguenga C, Carvalho F, Mansson JE. The g.1170C>T polymorphism of the 5' untranslated region of the human alphagalactosidase gene is associated with decreased enzyme expression- evidence from a family study. J Inherit Metab Dis. 2008;31 Suppl 2:S405-13. 
34. Poliakova AA, Gudkova AYA. P989. Association of acroparesthesias and angiokeratomas with the alphagalactosidase A gene polymorphisms in females with hypertrophic cardiomyopathy. European Heart Journal. 2019; 40 (1) ehz747.0494, https://doi.org/10.1093/eurheartj/ehz747.0494.

35. Ferreira S, Reguenga C \& Oliveira JP. The Modulatory Effects of the Polymorphisms in GLA 5'Untranslated Region Upon Gene Expression Are Cell-Type Specific. JIMD reports. 2015; 23, 27-34. https://doi.org/10.1007/8904_2015_424.

36. Schelleckes M, Lenders M, Guske K, et al. Cryptogenic stroke and small fiber neuropathy of unknown etiology in patients with alpha-galactosidase A -10T genotype. Orphanet J Rare Dis. 2014; 9:178. doi:10.1186/s13023-014-0178-5.

37. Michaud L. Longitudinal study on ocular manifestations in a cohort of patients with Fabry disease. PloS one, 2019;14(6), e0213329. https://doi.org/10.1371/journal.pone.0213329.

38. Gervas-Arruga, J., Cebolla, J. J., Irun, P., Perez-Lopez, J., Plaza, L., Roche, J. C., Capablo, J. L., RodriguezRey, J. C., Pocovi, M., \& Giraldo, P. Increased glycolipid storage produced by the inheritance of a complex intronic haplotype in the a-galactosidase A (GLA) gene. BMC genetics. 2015; 16,109. https://doi.org/10.1186/s12863-015-0267-z.

39. Alcalay RN, Wolf P, Levy OA, et al. Alpha galactosidase A activity in Parkinson's disease. Neurobiol Dis. 2018; 112:85-90. doi:10.1016/j.nbd.2018.01.012.

40. Pisani A, Imbriaco M, Zizzo C, Albeggiani G, Colomba P, Alessandro R, lemolo F \& Duro G. A classical phenotype of Anderson-Fabry disease in a female patient with intronic mutations of the GLA gene: a case report. BMC cardiovascular disorders. 2012; 12, 39. https://doi.org/10.1186/1471-2261-12-39

\section{Tables}

Table 1: Frequency of subtypes, clinical, laboratorial and drugs used in JIA patients: 


\begin{tabular}{|ll|}
\hline Features & $\mathbf{N}(\%)$ \\
\hline JlA subtypes: & \\
\hline Pauciarticular & $42(47.20)$ \\
Poliarticular RF Negative & $17(19.10)$ \\
Enthesitis related & $15(16.90)$ \\
Systemic & $10(11.20)$ \\
\hline Poliarticular RF Positive & $3(3.36)$ \\
\hline Psoriatic & $1(1.12)$ \\
\hline Indeterminate & $1(1.12)$ \\
\hline Symptoms: & \\
\hline Acroparesthesia & $47(52.80)$ \\
\hline Difficulty gaining weight & $30(33.70)$ \\
\hline Heat Intolerance & $26(29.50)$ \\
\hline Hyperhidrosis & $22(24.70)$ \\
\hline Dyspepsia & $19(21.30)$ \\
\hline Tinnitus & $18(20.20)$ \\
\hline Peripheric Neuropathy & $17(19.10)$ \\
\hline Abdominal distention & $14(15.70)$ \\
\hline Chronic fatigue & $14(15.70)$ \\
\hline Diarrhea & $11(12.40)$ \\
\hline Dysacusis & $8(9.00)$ \\
\hline Gastric fullness sensation & $6(6.74)$ \\
\hline Angiokeratoma & $2(2.24)$ \\
\hline Telangiectasia & 00 \\
\hline Family History: & $11(12.40)$ \\
\hline Stroke & \\
\hline Sudden death & \\
\hline kidney failure & \\
\hline Transient attack & \\
\hline Fabry & \\
\hline
\end{tabular}

Page 16/19 


\begin{tabular}{|ll|}
\hline LNA & \\
\hline Microalbuminuria (66 patients) & $9(12.32)$ \\
\hline HLA-B27 & $6(6.74)$ \\
\hline Rheumatoid Factor & $4(4.49)$ \\
\hline Anti-RNP antibody & $1(1.12)$ \\
\hline
\end{tabular}

Table 2: Cardiac Abnormalities in JIA Cohort and subtype descriptions - 74 patients - 20 cardiac abnormalities occurrence in $17(22.90 \%)$ patients.

\begin{tabular}{|c|c|c|}
\hline Features & Total N (\%) & Presentation $\mathrm{x}$ JIA Subtype \\
\hline \multirow[t]{4}{*}{ Right bundle branch block } & & 4 polyarticular \\
\hline & $10(52.63)$ & 1 systemic \\
\hline & & 3 oligoarticular \\
\hline & & 2 Entesitis-related \\
\hline \multirow[t]{2}{*}{ Mitral Valvar Prolapse } & & 1 polyarticular \\
\hline & $4(20)$ & 2 oligoarticular \\
\hline \multirow[t]{3}{*}{ Ventricular Hypertrophy } & & 1 polyarticular -VE (conc.) \\
\hline & $3(15.78)$ & 1 systemic - VD \\
\hline & & 1 Oligoarticular + FD - VE (conc.) \\
\hline \multirow{3}{*}{ Valvar regurgitation: } & & Mitral: 1 Oligoarticular + FD \\
\hline & $3(15.78)$ & Pulmonary: 1 Systemic \\
\hline & & Tricuspid: 1 Systemic \\
\hline
\end{tabular}

Legends: Conc: concentric

Table 3: Statistic correlation ( $\mathrm{p}$ value) between genetic variants and $\mathrm{ClHs}$ and clinical signs of Fabry disease, significant $p<0.05$. 


\begin{tabular}{|c|c|c|c|c|c|c|c|c|c|}
\hline Symptoms & $\begin{array}{l}\text { c. } 1000- \\
22 C>T\end{array}$ & $\begin{array}{l}\text { c.370- } \\
81 \_-77 d e l\end{array}$ & $\begin{array}{l}\text { c.640- } \\
16 \text { A> } \\
\text { G }\end{array}$ & $\begin{array}{l}c .-10 \\
c>T\end{array}$ & $\underset{T>C}{c 1244}$ & $\begin{array}{l}c .38 \\
C>T\end{array}$ & Hap1 & Hap2 & Нар3 \\
\hline AlfaGal Abn. & 0.624 & 1.000 & 1.000 & 0.429 & 0.073 & 0.927 & 1.000 & 1.000 & 1.000 \\
\hline GB3 Abn & 1.000 & 0.501 & 0.501 & 0.240 & 0.036 & 0.964 & 0.448 & 0.448 & 1.000 \\
\hline Heat Intolerance & 0.012 & 0.022 & 0.022 & 0.116 & 0.091 & 0.091 & 0.098 & 0.098 & 0.325 \\
\hline fatigue & 0.756 & 1.000 & 1.000 & 0.531 & 0.236 & 0.764 & 0.719 & 0.719 & 1.000 \\
\hline Tinnitus & 0.202 & 1.000 & 1.000 & 0.531 & 0.236 & 0.764 & 0.719 & 0.719 & 0.234 \\
\hline Dysacusis & 0.643 & 1.000 & 1.000 & 0.492 & 0.909 & 1.000 & 1.000 & 1.000 & 0.325 \\
\hline Acroparesthesias & 0.278 & 0.775 & 0.775 & 0.437 & 1.000 & 0.473 & 0.764 & 0.764 & 0.613 \\
\hline Hyperhidrosis & 0.222 & 0.102 & 0.102 & 0.624 & 0.273 & 0.273 & 0.493 & 0.493 & 1.000 \\
\hline Abdominal Dist & 0.745 & 0.730 & 0.730 & 0.519 & 0.218 & 0.782 & 1.000 & 1.000 & 1.000 \\
\hline Dyspepsia & 1.000 & 0.478 & 0.478 & 0.571 & 0.200 & 0.800 & 0.709 & 0.709 & 1.000 \\
\hline Diarrhea & 0.718 & 0.212 & 0.212 & 0.733 & 0.145 & 0.145 & 0.405 & 0.405 & 1.000 \\
\hline Weight Gain Diff & 0.775 & 0.213 & 0.213 & 0.024 & 0.309 & 0.691 & 0.322 & 0.322 & 1.000 \\
\hline Angiokeratoma & 0.436 & 0.291 & 0.291 & 0.127 & 0.018 & 0.982 & 0.255 & 0.255 & 1.000 \\
\hline Valvar Abn & 0.132 & 0.419 & 0.419 & 0.308 & 0.186 & 0.814 & 0.217 & 0.217 & 0.031 \\
\hline Arrhythmias & 1.000 & 0.602 & 0.602 & 0.465 & 1.000 & 0.907 & 0.572 & 0.572 & 1.000 \\
\hline Visual Changes & 0.073 & 0.046 & 0.046 & 0.005 & 0.213 & 0.787 & 0.01 & 0.01 & 0.521 \\
\hline Corneal Changes & 0.002 & 0.003 & 0.003 & 0.012 & 0.255 & 0.401 & 0.05 & 0.05 & 1.000 \\
\hline Cornea Vertic & 0.070 & 0.208 & 0.208 & 0,292 & 0.064 & 0.064 & 1.000 & 1.000 & 0.183 \\
\hline Cataract & 0.426 & 1.000 & 1.000 & 0.894 & 1.000 & 0.979 & 1.000 & 1.000 & 0.064 \\
\hline Ant. Chamb Ch & 0.004 & 0.006 & 0.006 & 0.011 & 1.000 & 0.128 & 0.164 & 0.164 & 0.343 \\
\hline Stroke FH & 0.184 & 0.565 & 0.565 & 0.645 & 0.436 & 0.436 & 0.756 & 0.756 & 0.307 \\
\hline Trans. attack FH & 0.186 & 0.081 & 0.081 & 0.240 & 0.036 & 0.001 & 0.448 & 0.448 & 1.000 \\
\hline Sudden death FH & 1.000 & 0.346 & 0.346 & 0.586 & 0.327 & 0.673 & 0.510 & 0.510 & 1.000 \\
\hline kidney failure FH & 0.686 & 0.660 & 0.660 & 0.423 & 1.000 & 0.891 & 1.000 & 1.000 & 0.379 \\
\hline Fabry FH & 0.436 & 0.291 & 0.291 & 0.127 & 0.018 & 0.982 & 0.255 & 0.255 & 1.000 \\
\hline
\end{tabular}

Legend: Hap 1= c.-10C>T, c.370-77_-81del, c.640-16A>G, c.1000-22C>T, Hap 2- c.370-77_-81del, c.640-16A>G, c. 1000-22C>T, Hap 3= c.548-125C>G, c. 1000-22C>T, c.-12G>A. Abn: abnormal, Dist: Distention, Diff: difficulty, cornea Vertic: cornea verticillata, Ant. Chamb Ch: Anterior chamber changes, , FH: Family history. 
Page 19/19 